\title{
Ad Hoc and Neighborhood Search Methods for Placement of Mesh Routers in Wireless Mesh Networks
}

\author{
Fatos Xhafa \\ Department of Languages and Informatics Systems \\ Technical University of Catalonia \\ Campus Nord, C/Jordi Girona 1-3 \\ 08034 Barcelona, Spain \\ EMail: fatos@lsi.upc.edu \\ Leonard Barolli \\ Department of Information and Communication Engineering \\ Fukuoka Institute of Technology \\ 3-30-1 Wajiro-higashi, Higashi-ku \\ Fukuoka 811-0295, Japan \\ EMail: barolli@fit.ac.jp
}

\author{
Christian Sánchez \\ Department of Languages and Informatics Systems \\ Technical University of Catalonia \\ Campus Nord, C/Jordi Girona 1-3 \\ 08034 Barcelona, Spain \\ EMail: csanchez@lsi.upc.edu
}

\begin{abstract}
With the fast development in wireless technologies and wireless devices, Wireless Mesh Networks (WMN) are becoming and important networking infrastructure, especially due to their low cost of deployment and maintenance. A main issue in deploying WMNs is the optimal placement of mesh routers to achieve network connectivity and stability through the maximization of the size of the giant component in the network and user coverage. In this work we evaluate ad hoc and neighborhood search methods for placement of mesh routers in WMNs. Routers are assumed having their own coverage area, oscillating between minimum and maximum values. Given a deployment area where to distribute the mesh router nodes and a number of fixed clients a priori distributed in the given area, ad hoc methods explore different topologies such as placement in diagonals, in corners of the area, in hotspots, etc. and the resulting network connectivity and user coverage are measured. We have experimentally evaluated the considered ad hoc methods through a benchmark of generated instances as stand alone methods and as initializing methods of evolutionary algorithms. For each ad hoc method, different distributions of mesh clients (Normal, Exponential and Weibull), are considered and the size of the giant component and user coverage parameters are reported. Further, we considered neighborhood search methods for optimal placement of mesh routers as more powerful methods for achieving near optimal placements of mesh router nodes. The experimental evaluation showed the good performance of a swap-based movement neighborhood search, which achieved good connectivity of the network in few phases of neighborhood search exploration.
\end{abstract}

\section{Introduction}

Wireless Mesh Networks (WMNs) [1] are becoming an important networking infrastructure due to their low cost and increased high speed wireless Internet connectivity. WMNs are special cases of ad hoc networks and are made up of wireless nodes organized in a mesh topology. Wireless nodes can be mesh routers, mesh clients and gateways. Mesh routers are interconnected by wireless links and provide Internet connectivity for mesh clients. WMNs are based on multi-hop communication paradigms -nodes can connect to each other through multiple hops.

Fast development of WMNs is pushed by their low cost nature that makes them an economical alternative for providing wireless Internet connectivity, especially in developing countries, avoiding costs of deployment and maintenance of wired Internet infrastructures. Applications of WMNs include WMNs for urban areas, community networking, metropolitan area networks, municipal wireless mesh networks, corporative networks, medical systems, transport systems, surveillance systems, etc. [2]. In all these applications, WMNs provide cost-efficient broadband wireless Internet connectivity to a group of users.

Mesh network topology distinguishes for providing reliability, robustness, and self-configuring properties achieved through multiple redundant communications paths in the network. The placement of mesh nodes plays an important role in achieving such properties. Indeed, the performance of WMNs is primarily affected by the location of mesh nodes, specifically, that of mesh router nodes of the WMN. However, in a real deployment of WMN the automatic or purely random node placements produce poor performance 
WMN since the resulting placement could be far from optimal. Further, real deployment of WMNs may require to take into account specific restrictions and characteristics of real geographic area and therefore one needs to explore different topologies for placing mesh routers. In fact, node placement can be seen as a crucial design and management issue in WMNs.

Mesh node placement in MWN can be seen as a family of problems. Different versions of the problem can be obtained depending on the types of mesh nodes to deploy as well as the objectives to optimize. For instance, in [6], [10], [8] is considered the gateway placement aiming to optimize the throughput. In [3], the authors consider a bi-objective version of the problem for two-tier WMNs.

Node placement belongs to the family of placement problems, which are shown (through graph theoretic approaches or placement problems, e.g. [4], [5]) computationally hard to solve for most of the formulations [11]. In fact, the node placement problem considered here is even more challenging due to two additional characteristics: (a) locations of mesh router nodes are not pre-determined (any available position in the considered area can be used for deploying the mesh routers), and (b) routers are assumed to have their own radio coverage area.

In this work we consider the version of the problem that given an area where to distribute a number of mesh router nodes and a number of mesh client nodes of fixed positions (of an arbitrary distribution), finds a location assignment for the mesh routers that maximizes the network connectivity (size of the giant component) and client coverage. These two objectives are among most important objectives in MWNs. Both of them are related to the performance of the network; the later can be also seen as a QoS in WMNs.

We consider approaching the mesh router nodes placement using ad hoc and neighborhood search methods; the former explore different topologies of the area such as node placement in diagonals, in corners of the area, in hotspots, etc.; in the later, neighbor solutions are explored in search for better placement of mesh routers. In both cases, the network connectivity and user coverage are measured and evaluated. Although in general using regular patters need not to provide optimal placement of nodes, ad hoc methods can be useful not only for concrete grid areas where to deploy mesh routers of a WMN but also as initializing methods for more powerful methods such as Genetic Algorithms. Moreover, we consider neighborhood search methods for near optimal placement of mesh routers as more powerful methods than ad hoc methods for achieving near optimal placements of mesh router nodes. In this case, the interest is to see how fast (in terms of phases of neighborhood search exploration) is achieved a good connectivity of the network.

We have experimentally evaluated the ad hoc and neighborhood search methods through a benchmark of generated instances. For each ad hoc placement method for mesh routers, different distributions of mesh clients (Normal, Exponential and Weibull), are considered and the size of the giant component and user coverage parameters are reported and evaluated. For the neighborhood search method, a swapbased movement is compared against a purely random search exploration.

The rest of the paper is organized as follows. In Section 2, we define the version of the problem under study. The considered ad hoc methods are presented in Section 3. In Section 4 we present neighborhood search methods. The experimental evaluation is given in Section 5. We end the paper in Section 6 with some conclusions.

\section{Problem Definition}

We consider the version of the mesh node placement problem in which, given an area where to distribute a number of mesh router nodes and a number of mesh client nodes of fixed positions (of an arbitrary distribution), finds a location assignment for the mesh routers that maximizes the network connectivity (size of the giant component) and client coverage. An instance of the problem consists of:

- $N$ mesh router nodes, each having its own radio coverage, defining thus a vector of routers.

- An area $W \times H$ where to distribute $N$ mesh routers. Positions of mesh routers are not pre-determined.

- $M$ client mesh nodes located in arbitrary points of the considered area, defining a matrix of clients.

The objective is to place mesh router nodes in points of considered area to maximize network connectivity and user coverage. In this work, the network connectivity is measured through the size of the giant component in the MWN. Network connectivity and user coverage are among most important metrics in WMNs. The former measures the degree of connectivity of the mesh nodes while the later refers to the number of mesh client nodes connected to the MWN. Both objectives are important and directly affect the network performance; nonetheless, network connectivity is considered as more important than user coverage. It should also be noted that in general optimizing one objective could effect the other objective although there is no direct relation among these objectives nor are they contradicting.

Notice from the above definition that mesh client nodes can be arbitrarily situated in the given area. For evaluation purposes, it is interesting, however, to consider concrete distributions of clients. For instance, it has been shown from studies in real urban areas or university campuses that users (client mesh nodes) tend to cluster to hotspots. Therefore different client mesh nodes distributions should be considered, for instance Weibull distribution, in evaluating MWN metrics.

We have considered Uniform, Normal, Exponential and Weibull distributions for client mesh nodes in the experimental evaluation (see Section 5). 


\section{Ad hoc Methods for Mesh Router Nodes Placement}

Ad hoc methods for placement of mesh routers are simple methods that explore different possible placement topologies. Their usefulness is two fold: (a) enabling fast computation of simple solutions for mesh router nodes placement, and (b) initializing other more sophisticated methods such as evolutionary algorithms that use population of solutions. We have consider seven ad hoc methods, namely: 1) Random, 2) ColLeft, 3) Diag, 4) Cross, 5) Near, 6) Corners, and 7) HotSpot. We briefly describe them next. It should be noted that in all considered methods, there is a pattern in placement of mesh router nodes, meaning that most of the node placements follow the pattern.

Random placement. In this method, mesh router nodes are uniformly at random distributed in the grid area.

ColLeft placement. This method places almost all mesh routers at the left side of the grid area. Some mesh routers could be placed at other parts of the grid area. The method is usually applicable when the number of mesh routers is (proportionally) smaller than grid area height, for instance, one third of the height.

Diagonal placement. In this method, mesh routers are concentrated along the (main) diagonal of the grid area. Again, this method is appropriate when the grid area fulfils some conditions such as the height and width must have similar values (we considered the case of $10 \%$ difference in their values) so that we can trace the diagonals.

Cross placement. This method tends to place mesh routers along both diagonals of the grid area. Similar conditions as the ones for Diagonal placement are required to ensure applicability of the method.

Near placement. In this method mesh routers are concentrated in the central zone of the grid area. To apply the method, minimum and maximum (user specified) values are considered to trace a rectangle in the central part of the grid area; routers are distributed in the rectangle cells.

Corners placement. This method distributes the mesh routers in the corners of the grid area. The considered areas in the corners are fixed by user specified parameter values.

HotSpot placement. This method starts by placing the most powerful mesh router in the most dense zone (in terms of client nodes) of the grid area; next, the second most powerful mesh router is placed in the second most dense zone, and so on until all routers are placed. This method seems particularly suited when distribution of mesh clients is not known a priori. It should be noted however that this method has a greater computational cost as compared to other methods due to the computation of denseness property.

\section{Neighborhood Search-based Algorithms}

The ad hoc methods presented in the previous section explore simple topologies for the placement of the mesh router nodes in the grid area. However, their solutions could be far from optimal ones. Algorithms based on neighborhood exploration are simple yet more powerful than ad hoc methods. The main idea is exploring the neighborhood of an initial solution by means of local moves and iterate until a stopping condition is met (for instance, there is no further improvement on the quality of solution.)

Different neighborhood search algorithms can be obtained depending on neighborhood structure, the acceptance criteria of next solution, etc. We present the pseudo-algorithm of neighborhood search in Algorithm 1. Starting from an initial solution, the algorithm first selects a movement type, that is the way the small local perturbation is performed, which defines the neighborhood structure. Then, iteratively, the algorithm computes the best neighbor of the current solution, namely, the best solution in the neighborhood of the current solution. If the best neighbor improves fitness of current solution, the current solution is moved to the best neighbor and so on.

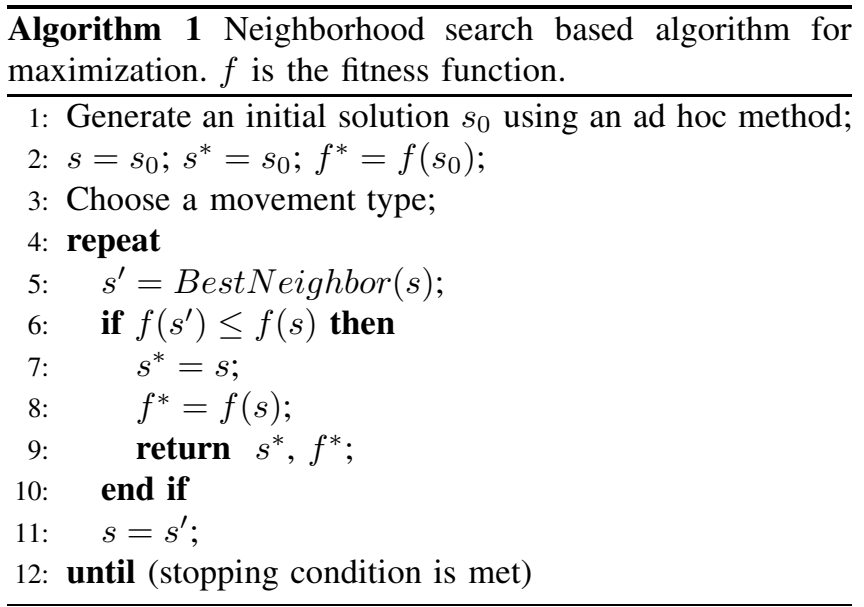

The computation of the best neighboring solution is presented in Algorithm 2. It should be noted that the exploration of the neighborhood can be done in different ways. For instance, we can systematically generate all movements, and hence, examine all possible solutions in the neighborhood; or, in case of large neighborhoods, just a pre-fixed number of movements is generated and corresponding neighboring solutions are examined.

Movement type can be defined in different ways. We considered the swap movement that consists in exchanging 


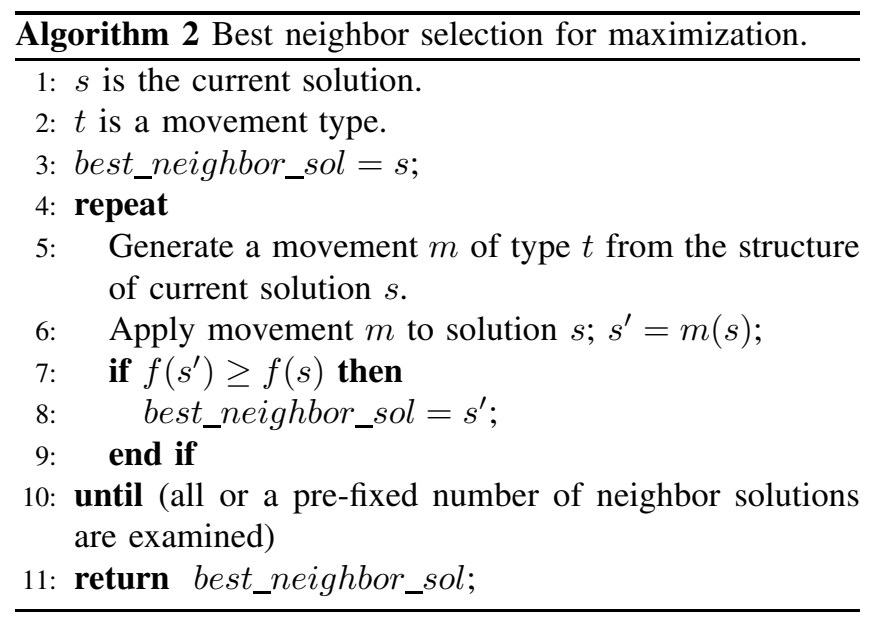

the placement of two routers. More precisely, the worst router (that of smallest radio coverage) in the most dense area is exchanged with the best router (that of largest radio coverage) of the sparsest area (see steps in Algorithm 3). The idea is to promote the placement of best routers in most dense areas of the grid area.

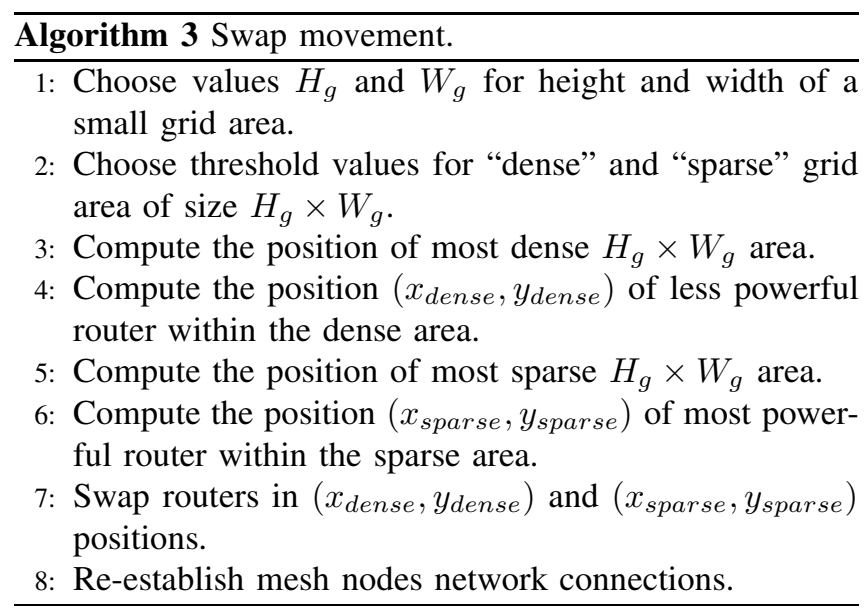

\section{Experimental Study}

The experimental evaluation is done in two steps. In the first, ad hoc methods are evaluated as stand alone methods. In the second one, ad hoc methods are evaluated by using a genetic algorithm implementation for the problem. In the former, the objective is to see the quality of placement of mesh routers regarding size of giant component and user coverage. In the later, ad hoc methods are used for generating the initial population of GA. The interest in this later case is to see the diversity of solutions provided by different ad hoc methods as initializing methods for evolutionary algorithms. In such algorithms, the diversity of the population, achieved through genetic information encoded in the chromosomes, is a crucial factor to avoid premature convergence of the algorithm to local optima. In the evolutionary algorithm literature there has been shown evidence that the quality of the initial solution plays an important role for the effective search of the algorithm. In this context, using ad hoc methods is more effective than pure random generation of initial population.

For the evaluation of neighborhood search, swap and random movement search are compared.

\subsection{Experimental setup}

We evaluated the proposed ad hoc methods through generated instances. Client mesh node positions were generated using four distributions, namely, Uniform, Normal, Exponential and Weibull. Instances were generated using these distributions and for each of them the size of the giant component and user coverage were measured. The objective is to see the quality of the ad hoc methods against different possible distributions of client mesh nodes in the grid area.

\subsection{Computational results}

In the next subsections we present computational results for ad hoc methods and neighborhood search algorithm.

5.2.1. Evaluation of ad hoc methods. We considered two scenarios for the evaluation of the ad hoc methods. In the first, we evaluated ad hoc methods as stand alone methods. In the second, we evaluated the quality of ad hoc methods as initializing methods of genetic based algorithms. Recently, there has been research interest in using evolutionary algorithms, such as Genetic Algorithms, for optimizing node placement and configuration (e.g. [9]). The interest in this later case is to see how does the quality of ad hoc methods influences the search efficiency and convergence of genetic algorithms.

Computational results for both scenarios are given in Tables 1, 2 and 3. Results in Table 1 correspond to an instance in which 64 routers are to be placed in a $128 \times 128$ grid area for covering 192 clients distributed according Normal distribution $N(\mu=64, \sigma=128 / 10)$.

The graphical representation of the evolution of size of giant component when ad hoc methods are used as initializing methods of GA is shown in Fig. 1. As can be seen from the figure, HotSpot is the best initializing method followed by Cross and Diag methods; ColLeft and Corners performed poorly.

Similarly, in Table 2 are given computational results for an instance corresponding to Exponential distribution. The graphical representation of the evolution of size of giant component when ad hoc methods are used as initializing methods of GA is shown in Fig. 2. As can be seen, HotSpot 
Table 1. Values of size of giant component and user coverage (client mesh nodes generated with Normal distribution.)

\begin{tabular}{|c|c|c|c|c|}
\hline Method & $\begin{array}{c}\text { Size of giant } \\
\text { comp. by GA } \\
\text { (ad hoc method) } \\
\text { initializing GA) }\end{array}$ & $\begin{array}{c}\text { User } \\
\text { coverage } \\
\text { by GA }\end{array}$ & $\begin{array}{c}\text { Size of giant } \\
\text { component } \\
\text { (ad hoc method } \\
\text { as stand alone) }\end{array}$ & $\begin{array}{c}\text { User } \\
\text { coverage } \\
\text { (ad hoc method } \\
\text { as stand alone) }\end{array}$ \\
\hline Random & 39 & 57 & 3 & 18 \\
\hline ColLeft & 35 & 52 & 8 & 3 \\
\hline Diag & 50 & 55 & 17 & 13 \\
\hline Cross & 54 & 74 & 13 & 19 \\
\hline Near & 48 & 60 & 13 & 35 \\
\hline Corners & 31 & 56 & 26 & 0 \\
\hline HotSpot & 64 & 86 & 4 & 10 \\
\hline
\end{tabular}

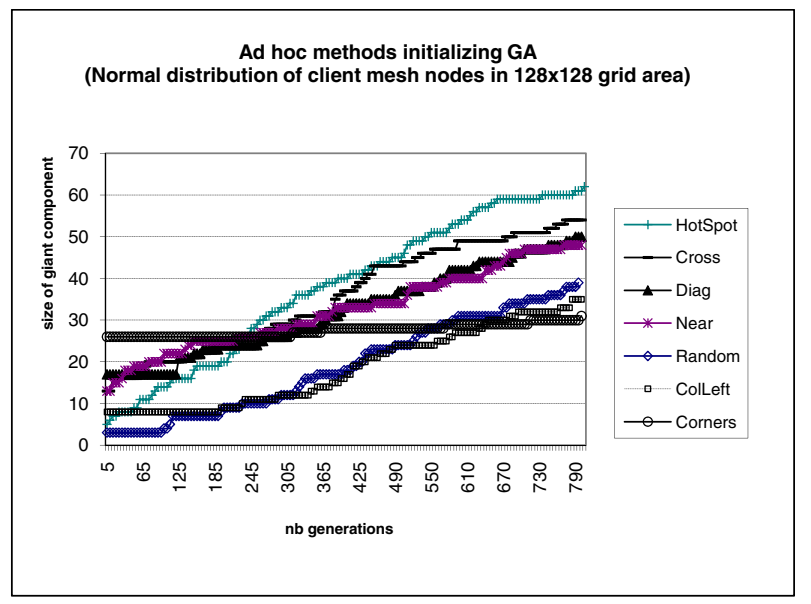

Figure 1. Evolution of size of giant component with Normal distribution of client mesh nodes (ad hoc methods used as initializing methods).

is the best initializing method followed by Cross and Diag methods; Corners and Random performed worst.

Finally, in Table 3 are given computational results for an instance corresponding to Weibull distribution. The graphical representation of the evolution of size of giant component when ad hoc methods are used as initializing methods of GA is shown in Fig. 3. Again, HotSpot is the best initializing method followed by Cross and Diag methods; Corners is the

Table 2. Values of size of giant component and user coverage (client mesh nodes generated with Exponential distribution).

\begin{tabular}{|c|c|c|c|c|}
\hline Method & $\begin{array}{c}\text { Size of giant } \\
\text { comp. by GA } \\
\text { (ad hoc method) } \\
\text { initializing GA) }\end{array}$ & $\begin{array}{c}\text { User } \\
\text { coverage } \\
\text { by GA }\end{array}$ & $\begin{array}{c}\text { Size of giant } \\
\text { component } \\
\text { (ad hoc method } \\
\text { as stand alone) }\end{array}$ & $\begin{array}{c}\text { User } \\
\text { coverage } \\
\text { (ad hoc method } \\
\text { as stand alone) }\end{array}$ \\
\hline Random & 29 & 97 & 3 & 32 \\
\hline ColLeft & 33 & 47 & 8 & 1 \\
\hline Diag & 54 & 27 & 17 & 11 \\
\hline Cross & 50 & 40 & 13 & 1 \\
\hline Near & 43 & 44 & 13 & 6 \\
\hline Corners & 26 & 18 & 26 & 8 \\
\hline HotSpot & 64 & 2 & 5 & 6 \\
\hline
\end{tabular}

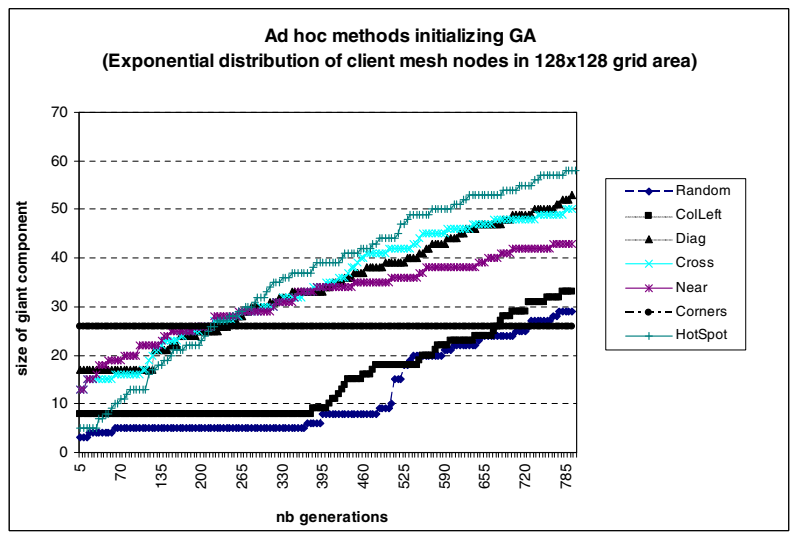

Figure 2. Evolution of size of giant component with Exponential distribution of client mesh nodes (ad hoc methods used as initializing methods).

worst one.

Table 3. Values of size of giant component and user coverage (client mesh nodes generated with Weibull distribution).

\begin{tabular}{|c|c|c|c|c|}
\hline Method & $\begin{array}{c}\text { Size of giant } \\
\text { comp. by GA } \\
\text { (ad hoc method) } \\
\text { initializing GA) }\end{array}$ & $\begin{array}{c}\text { User } \\
\text { coverage } \\
\text { by GA }\end{array}$ & $\begin{array}{c}\text { Size of giant } \\
\text { component } \\
\text { (ad hoc method } \\
\text { as stand alone) }\end{array}$ & $\begin{array}{c}\text { User } \\
\text { coverage } \\
\text { (ad hoc method } \\
\text { as stand alone) }\end{array}$ \\
\hline Random & 34 & 82 & 3 & 24 \\
\hline ColLeft & 33 & 67 & 8 & 12 \\
\hline Diag & 45 & 56 & 17 & 1 \\
\hline Cross & 46 & 62 & 13 & 3 \\
\hline Near & 45 & 41 & 13 & 0 \\
\hline Corners & 29 & 93 & 26 & 6 \\
\hline HotSpot & 63 & 10 & 4 & 12 \\
\hline
\end{tabular}

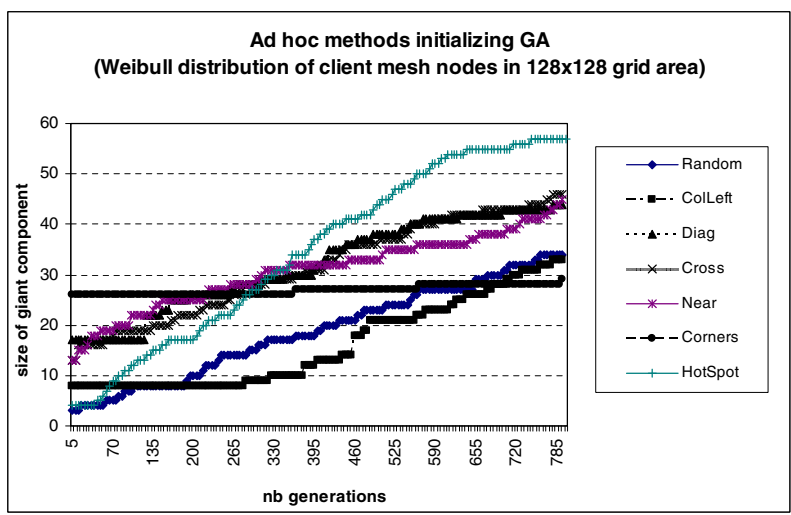

Figure 3. Evolution of size of giant component with Weibull distribution of client mesh nodes (ad hoc methods used as initializing methods).

5.2.2. Evaluation of neighborhood search. We compared the evolution of the neighborhood search for swap movement with that of a purely random movement for a $128 \times 128$ grid 


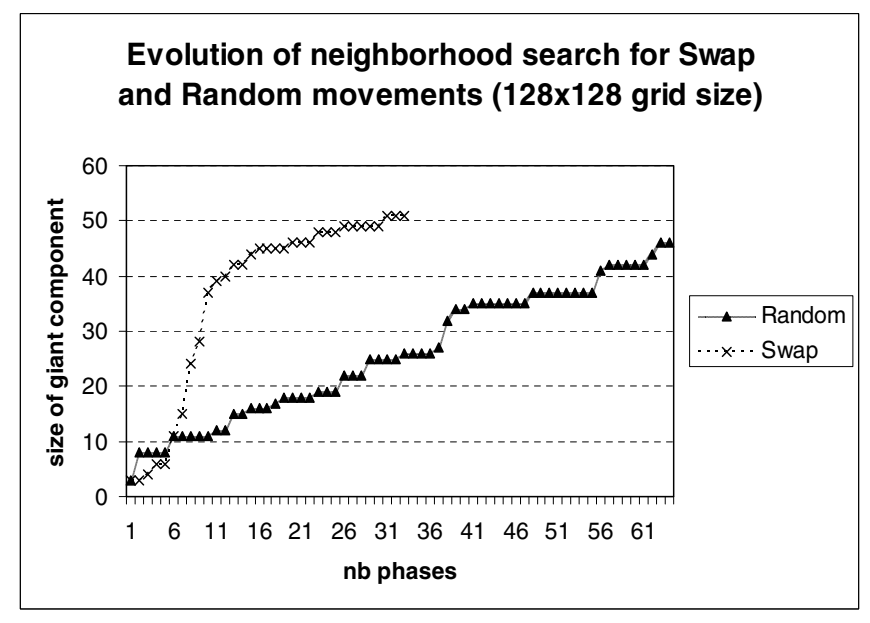

Figure 4. Evolution of size of giant component for neighborhood search.

area in which client mesh routers are distributed according to a normal distribution $N(\mu=64, \sigma=128 / 10)$. The graphical representation of the evolution of the search for both movements is shown in Fig. 4. As can be seen from the figure, swap movement achieves fast improvements on the size of the giant component.

\section{Conclusions}

In this work we have presented ad hoc and neighborhood search based methods for the problem of optimal placement of mesh router nodes in Wireless Mesh Networks (WMNs). We consider the version of the problem in which a number of client mesh nodes are a priori distributed in a grid area. The objective is to optimize the network connectivity and user coverage. Routers are assumed having their own radio coverage area.

The considered ad hoc methods explore different topologies in the grid area such as placement in diagonals, in corners of the area, in hotspots, etc. We experimentally evaluated the considered methods as stand alone methods as well as initializing methods of evolutionary algorithms. For each ad hoc placement method, different distributions of mesh clients (Normal, Exponential and Weibull) are considered and the size of the giant component and user coverage parameters are reported and evaluated. The ad hoc methods are very fast but have limitations in terms of optimality of the solutions. They showed however very useful as initializing methods of genetic algorithms. In this later case, the ad hoc method based on hotspot idea performed best.

Finally, we also considered neighborhood search based methods as more powerful methods than ad hoc methods. The experimental evaluation showed the good performance of a swap-based movement neighborhood search.
We are currently implementing full featured local search methods for the mesh router nodes placement.

\section{References}

[1] I. F. Akyildiz, X. Wang, and W. Wang. Wireless mesh networks: a survey. Computer Networks 47(4) (2005), 445-487.

[2] Ch. Chen and Ch. Chekuri. Urban Wireless Mesh Network Planning: The Case of Directional Antennas. Tech Report No. UIUCDCS-R-2007-2874, Department of Computer Science, University of Illinois at Urbana-Champaign, June 2007.

[3] A. Antony Franklin and C. Siva Ram Murthy. Node Placement Algorithm for Deployment of Two-Tier Wireless Mesh Networks. In Proceedings of IEEE GLOBECOM 2007, IEEE GLOBAL COMMUNICATIONS CONFERENCE, November 2007, Washington, USA, pp. 4823-4827.

[4] M.R. Garey and D.S. Johnson. Computers and Intractability -A Guide to the Theory of NP-Completeness. Freeman, San Francisco, 1979.

[5] A. Lim, B. Rodrigues, F. Wang and Zh. Xua. $k$-Center problems with minimum coverage. Theoretical Computer Science 332 (2005) 1-17.

[6] S. N. Muthaiah and C. Rosenberg. Single Gateway Placement in Wireless Mesh Networks. In Proceedings of 8th International IEEE Symposium on Computer Networks, Turkey, June 2008.

[7] N. Nandiraju, D. Nandiraju, L. Santhanama, B. He, J. Wang, and D. Agrawal. Wireless mesh networks: Current challenges and future direction of web-in-the-sky. IEEE Wireless Communications, 79-89 (2007).

[8] M. Tang. Gateways Placement in Backbone Wireless Mesh Networks. International Journal of Communications, Network and System Sciences, 1, 1-89. Published Online February 2009.

[9] T. Vanhatupa, M. Hännikäinen and T.D. Hämäläinen. Genetic Algorithm to Optimize Node Placement and Configuration for WLAN Planning. In Proceedings of 4th International Symposium on Wireless Communication Systems (ISWCS 2007), 612-616, 2007.

[10] P. Zhou, B. S. Manoj, and R. Rao. A gateway placement algorithm in wireless mesh networks. In Proceedings of the 3rd international Conference on Wireless internet (Austin, Texas, October 22 - 24, 2007).

[11] J. Wang, B. Xie, K. Cai and D.P. Agrawal. Efficient Mesh Router Placement in Wireless Mesh Networks, MASS 2007, October 9-11, 2007, Pisa, Italy. 